\title{
EL TELETÁNDEM EN EL APRENDIZAJE ALEMÁN - ESPAÑOL: MEDIATECA ENALLT - UNAM Y UNIVERSIDAD DE WÜRZBURG
}

\author{
Teletandem in German-Spanish Language Learning: \\ Mediateca ENALLT - UNAM and University of Würzburg
}

Edú Alberto Cruz LECONA

\author{
María Guadalupe ALFARO MARTÍNEZ²
}

\begin{abstract}
Resumen | El teletándem en la actualidad es un apoyo importante en el aprendizaje de una lengua extranjera, ya que este actúa como herramienta útil y eficiente desde el punto de vista del empleo de recursos prácticos. En el caso del español y del alemán, la Universidad de Würzburg y la Universidad Autónoma de México (UNAM) participan en un proyecto denominado Aprendizaje Virtual de Lenguas Extranjeras (AVLE). En este ámbito se evaluó la situación del desempeño de los estudiantes que aprenden español y de aquellos que aprenden alemán. La variable que se consideró en el rendimiento del aprendizaje es la confianza con la que los participantes pudieron resolver situaciones cotidianas, por ejemplo: ser capaces de comunicarse a través de pares en diferentes contextos culturales/ comunicativos: costumbres, fiestas nacionales y celebraciones típicas de sus países respectivos. A través de diarios de aprendizaje se pudo evaluar y reflexionar cómo fue el desarrollo de su aprendizaje. También se pudo advertir cómo fue su expectativa al inicio, durante el desarrollo y al final del proyecto. Por lo anterior, se pretende analizar la función de la mediateca de la Escuela Nacional de Lingüística Aplicada (ENALLT) y el Centro de Enseñanza de Lenguas Extranjeras de la Universidad de Würzburg.
\end{abstract}

Palabras clave | Teletándem. Centros de autoacceso. Mediateca. Würzburg. ENALLT.

\begin{abstract}
The teletandem is currently an important support in the learning of a foreign language, which has become a useful and efficient tool. The University of Würzburg and the Autonomous University of Mexico (UNAM) participate in a project called Virtual Learning of Foreign Languages (AVLE in Spanish). The performance of students who learn Spanish and those who learn German is evaluated. The variable that is seen in the learning performance is the confidence with which the participants adapt situations, for example: they work with information in different cultural / communicative contexts: customs, national holidays and typical celebrations of their countries. Through learning diaries, it was possible to evaluate and ponder how the development of their learning process was. It was also possible to announce how their expectations were at the beginning, during the development and at the end of the project. Therefore, we intend to weigh the role of the mediateca of the National School of Applied Linguistics (ENALLT) and the Foreign Languages Teaching Center of the University of Würzburg.
\end{abstract}

Keywords I Teletandem. Self-Access Center. Würzburg. ENALLT.

1 Lecona. UNAM. E-mail: edu_lecona@hotmail.com. ORCID ID: https://orcid.org/0000-0003-0761-207X 2 Alfaro Martínez. Würzbur Universität. E-mail: lupita_alfaro@web.de. ORCID ID: https://orcid.org/00000001-5268-6284 
- El teletándem en el aprendizaje Alemán - Español: mediateca ENALLT - UNAM y Universidad de Würzburg

\section{Introducción}

En este proyecto se presenta una evaluación cualitativa del resultado de las interacciones orales entre estudiantes universitarios de alemán y español nivel B1 en relación con el rendimiento del aprendizaje de dichas lenguas a través de las TIC (Tecnologías de la Información), como herramientas de comunicación y motivación en el aprendizaje de la lengua extranjera. El objetivo fue poner en práctica la utilización del programa Skype como medio de comunicación virtual fuera de las clases que se imparten en la institución para reforzar el aprendizaje de la lengua extranjera, para fomentar la autoevaluación, la autonomía, la confianza en sí mismo y la motivación del estudiante.

La interacción que ocurre en el teletándem es de tipo independiente, lo que facilitó a los participantes la libertad de actuar de manera más autónoma en cuanto al tiempo, lugar, hora y a la preparación de las sesiones.

Para la organización de los testimonios de los estudiantes, se utilizaron dos diarios de aprendizaje que contenían preguntas con problemas pragmáticos y sociolingüísticos que los llevó a una autorreflexión de su proceso de aprendizaje.

Los referentes teóricos en la presentación de este proyecto se apoyan en registros de experiencias pedagógicas en la enseñanza universitaria, realizadas en el contexto del desarrollo de habilidades orales (TARDO, 2005) y técnicas de la conversación (LORENZO A., DE COULOM, G 2005), así como en la definición de los tres principios teóricos fundamentales del teletándem (MENDOZA, 2014), (BRAMMERTS, 2006), (VASSALLO; TELLES, 2006):

\footnotetext{
- separación de lenguas: cada interacción en el teletándem se divide en dos partes y cada una de ellas debe estar dedicada a la práctica de una sola de las lenguas; - reciprocidad: los participantes deben turnarse como aprendices de una lengua extranjera y como expertos en su lengua materna (o lengua de competencia); - autonomía: cada participante debe decidir qué, cuándo, dónde, cómo y durante cuánto tiempo quiere aprender y debe evaluar su progreso. (VASSALLO, TELLES, 2006 apud CAVALARI; ARANHA, 2016, p. 328).
}

\footnotetext{
3 Teletandem practice is guided by three theoretical principles (VASSALLO; TELLES, 2006): - separation of languages: each teletandem interaction is composed of two parts and each one should be devoted to the practice of only one of the languages; - reciprocity: participants should take turns as the learner of a foreign language and the expert of their native language (or language of proficiency); - autonomy: each participant should decide what, when, where, how and how long they want to learn and should assess their progress.
} 
En investigaciones publicadas en XII Jornadas de redes de investigación en docencia universitaria (ICE de la Universidad de Alicante) en relación con las líneas teóricas generales del teletándem, se consultó particularmente a Chiapello, González y Mura (2014), para abordar los aspectos de planificación, realización y evaluación de los contenidos, en tanto que estrategias metacognitivas que se llevan a cabo al momento de la interacción para evaluar la propia cognición. El aspecto motivacional que conlleva la interacción de los participantes del teletándem, se define en las investigaciones de Boekaerts (GONZÁLEZ, 2014).

La perspectiva comunicativa e intercultural que implica este proyecto se encuadra en las líneas directivas de la política del Marco Común Europeo de Referencia para las Lenguas (CONSEJO DE EUROPA, 2002).

El objetivo del trabajo es describir la forma en que los participantes pueden resolver problemas en el momento de comunicarse a través de la estrategia teletándem. Así mismo, con base en diarios de aprendizaje se pretende que los estudiantes puedan plasmar en ellos sus experiencias en el momento de interactuary por ende, se intenta ver el rendimiento en ciertas áreas como la planificación, el manejo del léxico y el desarrollo de la confianza, además de tratar de describir cómo los estudiantes universitarios aprenden y comprenden aspectos culturales de México y Alemania.

\section{Marco conceptual}

El rendimiento en el aprendizaje siempre ha constituido uno de los desafíos frente al proceso de la enseñanza. La toma de conciencia y la responsabilidad del propio aprendizaje, así como el trabajo colaborativo, forman parte de las preocupaciones educativas que pretenden superar este reto. En lo que respecta al aprendizaje en la enseñanza de las lenguas extranjeras, estos aspectos forman parte de la política del Marco Común Europeo de Referencia para las Lenguas puesto que los "alumnos aprenden una lengua principalmente como miembros de una sociedad que tiene tareas (no solo relacionadas con la lengua) que llevar a cabo en una serie determinada de circunstancias, en un entorno específico y dentro de un campo de acción concreto" (CONSEJO DE EUROPA, 2002, capítulo 2.1.). Bajo este enfoque, el teletándem se centra en la acción de dos participantes nativos, quienes al momento de comunicar ponen en práctica sus competencias individuales, su conocimiento y su percepción del mundo, así como sus valores sociales. 
- El teletándem en el aprendizaje Alemán - Español: mediateca ENALLT - UNAM y Universidad de Würzburg

Chiapello (2014, p. 2080) observa que cuando se puso en marcha el Teletándem entre estudiantes de español e italiano se describieron las etapas del proceso de investigación: "organizar la interacción entre los estudiantes, recoger del corpus de interlengua en italiano como también en español y analizar y proponer criterios de evaluación."

Los diarios de aprendizaje que contestaron los estudiantes después de cada conversación y durante las conversaciones se enmarcan para su análisis en estas corrientes de investigación y en las orientaciones teóricas abordadas en estudios enfocados a la enseñanza de las lenguas extranjeras y al teletándem.

\section{Metodología del estudio}

\section{Contexto}

En el caso del Centro de Lenguas de la Universidad de Würzburg, la expresión oral ocupa un lugar importante entre los objetivos de los estudiantes de español quienes, después de un sondeo en uno de los cursos del nivel B1 del Marco Común Europeo de Referencia para las Lenguas (MCER) a través de un cuestionario en la primera clase del semestre, se constató que solo un estudiante tenía como primer objetivo aprender más de gramática. Para el resto del grupo, los objetivos principales fueron la comunicación oral y el conocimiento de otra cultura.

Una de las preocupaciones del departamento de español del Centro de Lenguas de la Universidad de Würzburg es cómo ofrecer más espacio a la práctica de esta habilidad en los cursos presenciales y aumentar, de esta manera, el rendimiento del aprendizaje del español. En este caso, el teletándem se trató como un proyecto piloto, y se ofreció a casi la mitad del grupo (seis participantes) la oportunidad de comunicar con nativos a través de las Tecnologías de la Información (TIC).

Por su parte, en la Mediateca de la Escuela Nacional de Lingüística y Traducción (ENALLT), el teletándem funciona como un proyecto en el cual, los participantes emplean esta metodología para poder ampliar su práctica en alguna de las lenguas extranjeras que se ofrecen en el centro de autoacceso y su desempeño es totalmente opcional. En este aspecto, los seis participantes aprendían alemán en el nivel B1 del MCER (Marco Común Europeo de Referencia para las Lenguas). 


\section{Tándem independiente}

La organización interna (horarios, parejas, diarios) se realizó a través de la mediateca de la ENALLT-UNAM. Se propuso una organización de tipo "tándem independiente" definido como un "contexto que mantiene un amplio espacio de libertad en términos de negociación y de responsabilidad por parte de los aprendientes" (MENDOZA, 2014, p. 7), puesto que el lugar y la hora se acordaron entre los mismos estudiantes debido a los husos de horario de México y de Alemania. El primer contacto se realizó por correo electrónico. El programa Skype fue el medio de comunicación que se empleó con más frecuencia por ser un medio que permite tener una conversación como si fuese "cara a cara" y al mismo tiempo se realiza una comunicación escrita a través del chat, el cual se usa como herramienta para enviar enlaces, comunicar dudas por escrito o para tomar notas.

\section{Herramientas}

Para lograr una mejor medición del rendimiento de los participantes, se proporcionaron dos "diarios de aprendizaje" como herramientas para recoger el trabajo y los comentarios de manera sistemática de las doce sesiones previstas y nos ofrecieron la posibilidad de poder organizar el resultado final de las sesiones de forma tanto individual como grupal. Se les dejó la libertad de escoger la lengua (español o alemán) para completarlos.

En estos "diarios de aprendizaje" hay una serie de preguntas abiertas a través de las cuales se pudieron dilucidar los recursos empleados por los participantes que los llevaron a reflexionar sobre una solución rápida a problemas pragmáticos, lingüísticos y socioculturales que surgieron durante las sesiones, además de promover la capacidad de autoevaluación y autorreflexión. Se explicó que no era necesario contestar todas las preguntas en todas las sesiones.

También se proporcionó a los participantes las top ten de sugerencias para optimizar las sesiones teletándem, en donde se recomiendan actividades de preparación tomando en cuenta los principios relacionados con el aprendizaje de lenguas extranjeras como la reciprocidad, la retroalimentación, así como consejos prácticos: tomar apuntes, usar el chat, usar una bitácora de aprendizaje.

Los temas propuestos tienen como objetivo promover la competencia sociocultural del estudiante, ya que son su interés y están relacionados con su vida cotidiana. También fomentan una conversación lo más espontánea posible y el diálogo auténtico. Se intercalan temas libres que ayudan a motivar a los participantes a abordar un tema de interés común. 
- El teletándem en el aprendizaje Alemán - Español: mediateca ENALLT - UNAM y Universidad de Würzburg

\section{Calendario, temas y sesiones}

Las sesiones se realizaron cada semana, normalmente con una duración de 60 minutos, alternando periodos de 30 minutos para utilizar la lengua meta y la lengua materna. La duración de las sesiones fue de cinco meses (semestre escolar de "invierno" 2017-18) con la participación de seis estudiantes de español nivel B1 en el caso del Centro de Lenguas de la Universidad de Würzburg y seis aprendientes de la mediateca de la ENALLT con nivel B1 en alemán.

\begin{tabular}{|c|c|}
\hline Focha do inicio & Tomas \\
\hline $\begin{array}{l}\text { Secion } 1 . \\
\text { Martes } 24 \text { de cx:tubre de } 2017 \text {. }\end{array}$ & Mi preeentacion/mi companero de tandem. Pasatiempoe, intereses y proyectoe. \\
\hline $\begin{array}{l}\text { Sesión } 2 . \\
\text { Martos } 31 \text { do octubro do } 2017 .\end{array}$ & $\begin{array}{l}\text { La Universidfad Autónoma de México (UNAM)/Universidad de Würzburg. ¿Qué es y cómo es mi } \\
\text { universided? }\end{array}$ \\
\hline $\begin{array}{l}\text { Sesion } 3 \text {. } \\
\text { Martes } 07 \text { de novinmben de } 2017 \text {. }\end{array}$ & $\begin{array}{l}\text { La Universidad Autonoma de Mexico (UNAM) Universidad de Würzburg II. ¿Por que es importante } \\
\text { para mi estudiar an mi universidad? ¿Qud me ofmece? }\end{array}$ \\
\hline $\begin{array}{l}\text { Snsibin } 4 \text {. } \\
\text { Martee } 14 \text { de noviemore de } 2017 .\end{array}$ & Lithen \\
\hline $\begin{array}{l}\text { Sesion 5. Martos } 21 \text { do novicmbro do } \\
2017 .\end{array}$ & Mi ciudad/ostado. ¿COmo son? ¿QUu atracciones mo ofrocon? \\
\hline $\begin{array}{l}\text { Sesión } 6 . \\
\text { Martos } 28 \text { do noviomoro do } 2017 .\end{array}$ & Costumbms y colebraciones en mi ciudadiestado. ¿Qub́ y cómo colnbramos? \\
\hline $\begin{array}{l}\text { Soaion } 7 . \\
\text { Martes } 05 \text { de clicierntbre de } 2017 \text {. }\end{array}$ & $\begin{array}{l}\text { Costumbros y colebraciones on mi ciudad/catedo II. i. Que hacemos los jovenos on nucstra ciudad } \\
\text { durante las vaciaciones? }\end{array}$ \\
\hline $\begin{array}{l}\text { Sessión } 8 \\
\text { Martos } 12 \text { do diciombro do } 2017 .\end{array}$ & 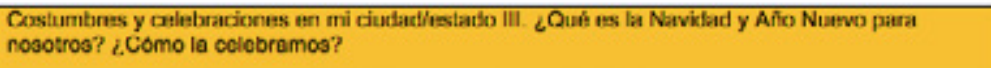 \\
\hline $\begin{array}{l}\text { Sesion } 9 \text {. } \\
\text { Martes } 09 \text { de cnero de } 2010 \text {. }\end{array}$ & Proyectos para el Ano Nuevo. ¿Que planeo realizar en el ano? \\
\hline $\begin{array}{l}\text { Sesión } 10 . \\
\text { Martee } 16 \text { de enero de 2018. }\end{array}$ & Libre. \\
\hline $\begin{array}{l}\text { Secion } 11 . \\
\text { Martes } 23 \text { den erern ile } 2018 .\end{array}$ & - $=$ \\
\hline $\begin{array}{l}\text { Sesión } 12 . \\
\text { Martes } 30 \text { de enero de } 2018 .\end{array}$ & Reflexiones finales. ¿Quó aprendl? ¿Quó experiencias tuve? \\
\hline
\end{tabular}

Figura 1. Temas y fechas sugeridos

\section{Perfil de los estudiantes}

Las parejas se organizaron por edades e intereses comunes. La edad de los estudiantes alemanes fue entre 19 y 25 años. Las carreras correspondientes fueron Medicina (2), Ciencias Políticas (2), Geografía, Economía y Medios de la Comunicación (2). En tanto para los participantes de la mediateca de la ENALLT la edad fue entre 18 y 19 años, de las carreras de Veterinaria (1), Ciencias Políticas (1), Letras Hispánicas (1), Física (1), Química (1) e Ingeniería (1). 


\title{
Análisis y evaluación de los diarios
}

González (2014, p. 1448) afirma que:

\begin{abstract}
Las estrategias meta-cognitivas hacen referencia a la planificación, control y evaluación por parte de los estudiantes de su propia cognición. Son un conjunto de estrategias que permiten el conocimiento de los procesos mentales, así como el control y la regulación de los mismos con el objetivo de lograr determinadas metas de aprendizaje. La misión fundamental de estas estrategias es perfeccionar la eficacia del aprendizaje mejorando las condiciones en las que se produce. Esas estrategias incluyen: establecery mantener la motivación, enfocar la atención, mantener la concentración, manejar la ansiedad y el tiempo de manera efectiva.
\end{abstract}

Un análisis cualitativo de los diarios de aprendizaje revela la progresión lograda en las funciones comunicativas y en las destrezas que los estudiantes practicaron durante las conversaciones para mantener el diálogo en los procesos de planificación, control y evaluación.

\section{Análisis diario de aprendizaje versión uno}

Aquí se analiza un primer diario de aprendizaje, cuyo objetivo es llevar al estudiante a adquirir mecanismos de autorregulación que "describen los distintos componentes que están implicados en el aprendizaje exitoso, explicar las relaciones recíprocas y recurrentes que se establecen entre ellos y relaciona el yo o, lo que es lo mismo, con las metas, la motivación, la volición y las emociones" (BOEKAERTS,1999 apud GONZÁLEZ, 2014, p. 1449). El diario se centra en elementos del proceso antes, durante y después de la conversación. De esta manera, el participante, de forma libre, prepara el vocabulario, las cosas y las frases que quiere decir y utilizar en la lengua extranjera. También propone anotar el vocabulario o las ideas que surgieron durante la conversación. Incluye igualmente una evaluación sobre el nivel de satisfacción de los participantes de su competencia lingüística en cuanto a vocabulario, gramática, pronunciación, fluidez, así como la confianza con la que enfrenta la interacción. 
- El teletándem en el aprendizaje Alemán - Español: mediateca ENALLT - UNAM y Universidad de Würzburg

Las tres primeras partes de este diario de aprendizaje abordan la planificación del léxico de la sesión, el monitoreo del mismo en los diferentes momentos de la conversación y la valoración del aprendizaje. La cuarta parte lleva a la autorreflexión del estudiante sobre su participación en la sesión y da la oportunidad de proponer cambios en futuras sesiones, lo cual constituye un aspecto importante en la progresión hacia su autonomía.

\section{Toma de responsabilidad}

\section{Planificación}

Este aspecto básico en la enseñanza de lenguas extranjeras se abordó en la fase de preparación y corresponde a la primera pregunta del diario, bajo la rúbrica "Prepara lo siguiente: vocabulario/cosas que quiero decir". Se pudo constatar en las declaraciones reunidas que la preparación del léxico de las sesiones de los estudiantes alemanes fue bastante sistemática. Algunas declaraciones muestran que, si se realiza una planificación de la sesión, el aprovechamiento tiene más éxito, como se observa en los fragmentos:

(1) Hoy me preparé muy bien y pude hablar bien. El tema fue tan interesante que pude darle a mi compañera una pequeña visión de Alemania.

(2) Fue importante preparar el tema de la conversación para no tener que consultar muchas palabras durante la misma.

Otro aspecto es la gestión del tiempo, la cual también fue más efectiva:

(3) Me preparé bien y tuve bastante materia para hablar durante mis 25 minutos.

Para los participantes de la ENALLT, la situación no se aleja de la descripción anterior, ya que ellos mismos planificaron sus sesiones en función de su tiempo y de la disponibilidad del vocabulario/léxico y lo que ellos deseaban expresar.

\section{Monitoreo del léxico}

En la segunda etapa del proceso, "Durante la conversación", se incita al estudiante a tomar nota de los aspectos relevantes de la misma con respecto al vocabulario o a las situaciones que surgieron en esa etapa. Las declaraciones de los estudiantes alemanes revelaron que la mayoría recuperó el vocabulario que consideró importante durante la 
conversación. En otras ocasiones, los estudiantes también resumieron el contenido de la conversación. Un estudiante alemán mostró su nivel de autonomía y su participación colaborativa, porque se hizo responsable del aprendizaje de su compañero además del mismo y en algunos casos lo hizo, incluso, con mucho empeño, porque se ponía en la posición del profesor al anotar los errores cometidos y al exponer la respuesta correcta.

Lo anterior se basa en las ideas que plasmaron los aprendientes de la ENALLT en sus diarios, ya que ellos dijeron sentirse apoyados por su contraparte alemana cuando necesitaban ayuda en el vocabulario que no conocían.

\section{Autovaloración}

En la tercera rúbrica del diario 1, "Después de la conversación" se aborda el proceso de la autovaloración y se mide en una escala del 1 al 5, desde "muy poco contento" hasta "mucho muy contento". Se ponen de relieve estrategias cognitivas que valoran aspectos de tipo lingüístico (pronunciación, fluidez, dominio del léxico y gramática) y motivacionales o de actitud ante la tarea que debe realizar: confianza en sus propias capacidades. Este último aspecto está en estrecha relación con los aspectos lingüísticos anteriores puesto que engloba las apreciaciones que tuvieron los estudiantes sobre su desempeño durante las conversaciones.

\section{Confianza}

En el caso de los estudiantes alemanes, se puede observar que no hubo cambios significativos individuales en el aspecto de la confianza en sí mismo. Desde el principio del teletándem, hasta el final de las sesiones se mantuvo constante la puntuación personal. El grado de confianza durante las conversaciones fue, en general, muy alto, puesto que ellos se encuentran en el nivel 5 de confianza. Este sentimiento de confianza en sí mismo se relaciona tanto con aspectos de la fase de la preparación ya evaluada en el "diario 1", así como con aspectos afectivos reflejados en el "diario 2", en donde se da la situación de comunicación con su compañero del teletándem. Por ello, los aprendientes de la ENALLT tampoco expresaron cambios importantes y eso está relacionado con el acompañamiento que sintieron con sus respectivas parejas en cuanto a la confianza para expresar sus ideas en los dos idiomas. 
- El teletándem en el aprendizaje Alemán - Español: mediateca ENALLT - UNAM y Universidad de Würzburg

\section{Vocabulario}

El vocabulario obtuvo una mayoría entre 2 y 4 puntos en los estudiantes alemanes. El grado de mayor satisfacción se mantuvo en la valoración 3 (contento), lo cual puede ponerse en relación con la etapa de preparación del vocabulario y con aspectos relacionados con las dificultades que aparecieron durante la conversación abordados en el diario 2. Esto también se constata en el aspecto gramatical, donde la puntuación fue en aumento en el transcurso de las sesiones y tuvo una fluctuación para la mayoría de los estudiantes alemanes entre 2 en las primeras sesiones y 3-4 puntos al final. El promedio general fue de 3 puntos (contento). Con base en lo anterior, para los seis jóvenes mexicanos también valoraron su desempeño de satisfacción entre 2 y 4 puntos. Algunos aprendientes de la ENALLT expresaron en sus diarios que sintieron que aprendieron mucho más vocabulario durante las sesiones, que en la etapa de preparación.

\section{Pronunciación}

En el dominio de la pronunciación se mantuvieron como media general las valoraciones 3 y 4 (contento-muy contento) durante las sesiones. En la última sesión se constató la mayor satisfacción con la apreciación 4 en el diario de los estudiantes alemanes. Es así como para los mexicanos, la valoración en la pronunciación fue muy alta. La relación entre confianza y mayor aprendizaje también se relaciona con la pronunciación.

\section{Fluidez}

La fluidez, junto con la capacidad de articular son definidos por el MCER (Marco Común Europeo de Referencia para las Lenguas) como "dos factores genéricos y cualitativos que determinan el éxito funcional del alumno o usuario para seguir adelante y desenvolverse bien cuando se llega a un callejón sin salida" (CONSEJO DE EUROPA, 2002, capítulo 5.3.2.). La fluidez comparte aspectos de "entonación-pronunciación aceptables; seguridad y convicción" (LORENZO, COULOMB, 2005, p. 113), y por lo tanto está en estrecha relación con los otros aspectos evaluados en esta tercera pregunta del diario. Entre los estudiantes alemanes se puede constatar que el resultado fue similar al de los aspectos analizados anteriormente, destacando la nota 3 (contento) en su evaluación. La valoración global final en cuanto a este aspecto, es muy positiva, como se puede observar en los relatos: 
(4) Pudimos comunicarnos con fluidez, lo cual me hizo sentir orgullosa.

(5) Me di cuenta que cada vez era más fácil para mí hablar, que hablaba con más fluidez y que cometía menos faltas.

(6) Tenía la sensación de que había aprendido a hablar con más fluidez y también descubrí que Raquel podía mejorar sus habilidades en el idioma alemán.

(7) Ahora puedo hablar con más espontaneidad y construir frases más rápidamente en español.

La pregunta número 4 del diario 1, "Piensa cómo fue el intercambio, qué cambiarías", muestra también aspectos enmarcados dentro del modelo del aprendizaje autorregulado. En el caso de los estudiantes alemanes se puede observar que en las respuestas que se obtuvieron, durante las primeras sesiones, los comentarios se concentraron sobre todo en las percepciones afectivas experimentadas durante el desarrollo de la sesión "conversación agradable", "nos entendimos muy bien" y "me alegro de conocerlo", "me dio mucho gusto conocerlo". En la medida en que la interacción entre los participantes se vuelve más frecuente, la confianza aumenta. También hubo comentarios que abordan metas a alcanzar dentro del aspecto lingüístico como llegar a tener una mejor fluidez o tomar nota para aprender el vocabulario "difícil" o desconocido.

\section{Autonomía}

Brammerts (2006, p. 2) define al espacio en el que el teletándem se desarrolla como un

\footnotetext{
[...] contexto de aprendizaje donde comunicary aprender en tándem son dos situaciones que llevan a los participantes a determinar su propio aprendizaje, donde cada uno de ellos puede decidir por sí mismo lo que quiere aprender y el tipo de ayuda que quiere recibir de su compañero. ${ }^{4}$
}

Este principio de autonomía se percibe, en el caso de los estudiantes alemanes, sobre todo en el deseo de abordar más temas libres. La motivación y los retos también son mayores en la preparación de estas sesiones. En uno de los testimonios se ve claramente

4 Zwei Personen mit unterschiedlichen Muttersprachen können voneinander lernen, denn beide verfügen schon über kulturelles Wissen und sprachliche Fertigkeiten, die der andere noch erwerben will. 
- El teletándem en el aprendizaje Alemán - Español: mediateca ENALLT - UNAM y Universidad de Würzburg

que el estudiante aprovecha la posibilidad de proponer, incluso, uno de los temas de las lecciones del curso de español y propone hablar sobre su infancia, "Cuando éramos pequeñas", en donde no solo abordó los tiempos del pasado, sino también todo el vocabulario relacionado con los juegos y pasatiempos de la infancia.

En los testimonios de dos participantes, que ya habían tenido una estancia en un país de habla hispana, se constató que solicitaron una mayor libertad en cuanto a los temas de conversación, como en los ejemplos:

(8) Los temas son muy parecidos.

(9) Hablamos también de otras cosas que nos interesaban.

(10) Tuvo un carácter muy forzado, porque siempre tenías que estar bien preparado y tener suficientes temas para mantener una conversación y a menudo los temas de los que hablamos se repetían.

Uno de ellos llegó a expresar su deseo por tener un diálogo presencial o de "cara a cara" por considerarlo más "espontáneo" para comunicar:

(11) Si os encontráis en persona y hacéis cosas juntos, entonces podéis reaccionar mucho mejor a las situaciones espontáneas y poneros en contacto aún mejor y hablar de cosas que no podéis abordar por Skype.

\section{Colaboración}

El principio de colaboración está en correlación con el principio de autonomía porque

[...] parece controlar los niveles de responsabilidad y poder que el hablante competente puede tener sobre el proceso de aprendizaje de su pareja. Los participantes nunca están solos en su proceso y cada uno de ellos puede ser apoyado y animado por su socio más competente en un esfuerzo de colaboración. (VASSALLO, TELLES, 2006, p. 89). ${ }^{5}$

5 The autonomy principle is relevant, because it seems to control the levels of responsibility and power that the proficient speaker may have over his/her partner's learning process. Tandem participants are never all alone in their process and each of them may be supported and encouraged by his/her more proficient partner in a collaborative endeavor. 
Este proceso representa uno de los aspectos de la conversación valorado muy positivamente durante todas las sesiones. La responsabilidad de ayudar al compañero a expresarse contribuye a mantener la dinámica del diálogo, abordando aspectos de vocabulario y gramaticales, como se nota en los testimonios:

(12) No tuvimos problema con el tema, sino con la gramática y vocabulario, pero fuimos pacientes y nos ayudamos mutuamente a expresarnos.

(13) El intercambio estuvo muy bien porque nos interesamos por lo que el otro dice. Nos corregimos las faltas y aclaramos lo que dijimos mal, por ejemplo, en los tiempos verbales.

(14) Muy bien, como siempre. Nos ayudamos mutuamente todo el tiempo.

(15) Hoy pudimos ayudarnos mutuamente cuando alguna de las dos no recordaba la palabra.

(16) No hubo ningún problema cuando el otro necesitaba más tiempo para expresarse. No hubo pausas incómodas por falta de palabras.

En la última sesión hubo comentarios como:

(17) Estuvo muy bien que los dos éramos igual de buenos, o malos en hablar la otra lengua, por esta razón, no nos daba vergüenza cometer errores o ayudar al otro con paciencia.

(18) Así hicimos conscientes nuestros puntos fuertes y nuestras deficiencias y las trabajamos juntos.

(19) Mi compañero de tándem y yo nos entendimos muy bien y nos ayudamos mutuamente a resolver los problemas.

\section{Motivación e Interculturalidad}

Tardo (2005, p. 3) considera que:

[...] la responsabilidad requiere una toma de decisiones por parte del alumno que aprende una lengua extranjera (LE), no sólo en cuanto a convertirse en protagonista de su propio proceso de adquisición, sino también debe ser valorado como una posición de autocontrol estratégico ante una necesidad inmediata de interacción comunicativa; lo que implica también un alto grado de motivación. 
- El teletándem en el aprendizaje Alemán - Español: mediateca ENALLT - UNAM y Universidad de Würzburg

Los datos cualitativos obtenidos en el diario 1 constatan que el aspecto motivacional está en estrecha relación con el aspecto intercultural. El intercambio aportó nuevas amistades. La mayoría de los participantes tiene la intención de seguir en contacto con su pareja del teletándem, con la perspectiva de viajar al país extranjero, visitarse o recibirla en su propio país:

(20) Espero poder visitar algún día México. Fue muy bonito que a través de este proyecto pudiera establecer un contacto.

(21) En el futuro vamos a tratar de comunicarnos y de escribirnos de vez en cuando. Mi compañero de teletándem vendrá a Alemania por algunos meses y lo visitaré.

(22) Estaba un poco triste porque era la última sesión del teletándem, pero nos pusimos de acuerdo en comunicarnos de vez en cuando por Skype para mantener el contacto.

El desarrollo de la competencia intercultural se propicia desde el principio a través de algunos temas propuestos en las sesiones y es un factor de motivación importante:

(23) Estuvo muy bien y es muy interesante saber cómo son otras tradiciones.

(24) Me pareció bien contarle las tradiciones alemanas en Año Nuevo, por ejemplo: "Dinner for one" o comer una "raclette".

También en los temas libres negociados entre ellos mismos se aborda el aspecto intercultural: "Día de los muertos/Oktoberfest", "Películas, series", "Lugares de interés en México y Alemania" o de tipo más personal como "Nuestra carrera", "Cumpleaños", "Cuando éramos pequeñas" (juegos tradicionales en cada país).

Al final del teletándem, en la sesión 12, todos los comentarios fueron muy positivos:

(25) El intercambio cultural fue muy interesante. Aprendí mucho sobre la cultura mexicana y la vida cotidiana. Espero poder visitar algún día México.

(26) Cada uno pudo hablar sobre su cultura.

(27) Creo que es bueno que este proyecto haya sido ofrecido, ya que nos dio la oportunidad de ponernos en contacto con México y practicar español.

(28) Los temas eran de actualidad y siempre fue interesante saber cómo son las tradiciones en México. 


\section{Motivación y aprendizaje}

En las apreciaciones en cuanto a la experiencia del teletándem y el grado de motivación que suscitó va unido también a la experiencia del aprendizaje y a la práctica de la lengua. La mayoría afirmó haber mejorado sus conocimientos sobre la lengua y haber adquirido una mayor fluidez y confianza al hablarla, como se ha podido constatar en las secciones anteriores. También se expresó la posibilidad de seguir en contacto para continuar aprendiendo la lengua extranjera:

(29) Podemos decir que jencontramos un buen amigo! Emiliano y yo queremos seguir en contacto para seguir mejorando mi español y él su alemán y así tener una persona a quién poder preguntarle nuestras dudas o para resolver problemas de la lengua.

(30) El teletándem fue para mí una experiencia extraordinaria, tuve un compañero de tándem muy bueno con el que aprendí mucho y tuve conversaciones hermosas.

(31) En general encuentro muy buena la idea de un intercambio entre estudiantes de Würzburg y estudiantes de México. Fue una muy buena oportunidad para aprender español además de las clases de español y consolidar aún más el idioma.

(32) Gracias a las conversaciones por Skype, perdí el miedo de hablar en español.

(33) En general pienso que el teletándem fue una muy buena idea, sobre todo para aprender una lengua extranjera.

(34) El teletándem fue para mí una buena oportunidad para mejorar mis conocimientos de español.

\section{Análisis diario de aprendizaje versión dos}

Este diario aborda preguntas que llevan a los estudiantes a reflexionar sobre sus destrezas y habilidades interactivas como la reciprocidad y el trabajo colaborativo, sobre su proceso de desarrollo personal (autoconocimiento, autoeficacia) y las estrategias del estudiante para resolver problemas. Las estrategias empleadas en esta fase del proceso de aprendizaje controlan o monitorean el rendimiento en el aprendizaje de la lengua extranjera. El resultado pone de manifiesto el grado de cooperación por las dos partes para establecer la comunicación. El análisis de las preguntas permite una sistematización de las respuestas y su ponderación. 
- El teletándem en el aprendizaje Alemán - Español: mediateca ENALLT - UNAM y Universidad de Würzburg

\section{Reconocimiento de emociones}

\section{Pregunta: Antes de iniciar la conversación me sentí...}

En el primer contacto en la primera sesión, las respuestas de los estudiantes alemanes se relacionan a sentimientos de nerviosismo e inseguridad (4/6) y de curiosidad (2/6). Una de estas dos últimas participantes se sintió "curiosa y segura", especificando que se debió a que se preparó para esta sesión con "apuntes". Los sentimientos de inseguridad fueron por el hecho de mantener por primera vez en español "una conversación completa" o por no saber si iban a darse a comprender en español. En la segunda sesión, el sentimiento de inseguridad disminuyó notablemente: "No tan nerviosa/crispada" (4/6), "muy relajado" (1) y solo una de las participantes se sintió "nerviosa", pero se "alegraba de la sesión" y la otra debido a la falta de preparación de la sesión. A partir de la tercera sesión se mantiene constante el sentimiento de seguridad. En algunas ocasiones esto se debe a la preparación de la sesión o a la motivación e al interés por volver a hablar con su compañero/a de teletándem.

\section{Pregunta: Cuando inició la conversación en alemán/español me sentí....}

La siguiente fase va relacionada con el hecho de comenzar la sesión en español o en alemán. En este aspecto, los participantes alternaron la lengua al principio de las sesiones. En la primera sesión el sentimiento de nerviosismo disminuyó: "más segura/aliviada" (2/4) "poco/algo nervioso" (2/4). La empatía que se estableció inmediatamente después del primer momento, aportó más seguridad "amabilidad y paciencia del compañero". En el segundo caso el sentimiento de inseguridad se debe al nivel de la lengua hablada por el compañero y la preocupación de comprensión: "no sabía el nivel de alemán de la compañera y tampoco si lo comprendía". Se constata también que a partir de la tercera sesión se estabilizan las opiniones de manera muy positiva variando entre muy bien y bien (31 veces en 12 sesiones) tomando en cuenta que una de las participantes nunca contestó a esta pregunta y que no era obligatorio contestar siempre a todas las preguntas. Hubo una opinión negativa solamente por falta de organización de los temas: “Un poco desbordado al iniciar la conversación en alemán porque ya se había hablado de muchos temas y no sabía cómo comenzar". Estas valoraciones van acompañadas a veces por comentarios complementarios como "contenta", "segura", "relajado", "acostumbrados".

De esta forma, se puede deducir que el sentimiento de seguridad está relacionado sobre todo con la confianza y la empatía que se establecen entre los participantes, así como con la rutina creada a través de las sesiones. 


\title{
Proceso de reflexión
}

Tardo (2005, p. 3) observa que:

\begin{abstract}
la reflexión permite al alumno convertirse en su propio crítico, evaluar el proceso, una idea o la solución a un determinado problema comunicativo. A partir del distanciamiento que se produce durante la reflexión se puede detectar, por parte del aprendiz, cuáles son los aspectos o áreas en las que presenta una mayor dificultad.
\end{abstract}

Pregunta: Cuando no encontraba las palabras o las frases para expresarme, yo....

La estrategia más utilizada por los estudiantes alemanes, en todas las sesiones para encontrar las palabras desconocidas o construir frases, fue parafrasear la palabra (25 veces) y consultar el diccionario por Internet (21 veces). Dos estudiantes solicitaron ayuda al compañero de manera sistemática pidiendo la traducción directa (alemán-español) (17 veces). Un estudiante expresó su sentimiento de frustración al no recordar la palabra en español.

Pregunta: Cuando no entendía lo que mi compañero me decía, yo ...

Se pudo constatar que, durante las doce sesiones, la combinación "repetir" la frase o la palabra (lentamente) se nombró (27 veces) y "parafrasear" (16 veces) fueron las estrategias más empleadas. La traducción directa de la palabra fue otro de los recursos, aunque poco utilizado (9 veces). También se utilizó la expresión escrita como recurso al momento de solicitar ayuda (escribir lo dicho), con menor frecuencia (7 veces).

Pregunta: ¿Qué dificultades tuve? ¿Cómo las solucioné?

La mayor parte de las dificultades encontradas a través de las sesiones para los estudiantes alemanes, se relacionan con la "falta de vocabulario" mencionada (26 veces), con la conjugación de los verbos (8 veces), variedad de expresiones (1 vez), variedad de temas (1 vez). Al analizar las estrategias utilizadas para resolver el problema, se corroboran las respuestas de la pregunta precedente. Entre ellas se nombra la utilización del diccionario (13 veces), la demanda de ayuda al compañero del teletándem, el parafraseo (4 veces). Con menor frecuencia se mencionó la utilización del inglés para explicar la palabra (2 veces). 
- | El teletándem en el aprendizaje Alemán - Español: mediateca ENALLT - UNAM y Universidad de Würzburg

Cuatro de las preguntas que finalizan el diario de aprendizaje 2 se relacionan con estrategias de tipo metacognitivo concernientes al proceso del desarrollo personal en el desempeño del aprendizaje de la lengua extranjera por parte de los participantes.

Pregunta: Creo que debo mejorar en....(reconocimiento de carencias).

En esta parte las respuestas se orientaron sobre todo a aspectos de gramática. Aprender los tiempos verbales se nombró (22 veces), los verbos y las preposiciones (3 veces), aprender más gramática en general (5 veces), más vocabulario (11 veces), fluidez (6 veces).

Pregunta: Me di cuenta que puedo....( reconocimiento de logros).

Las respuestas más frecuentes a esta pregunta se refieren al aspecto de la comprensión de lo que su compañero de teletándem dice durante la conversación en español (12 veces), expresarse bien (11 veces). Se hizo referencia generalmente al aspecto gramatical: expresarse bien en pasado, hacer las concordancias entre adjetivo y sustantivo, utilizar los "conectores" en sus frases. La toma de conciencia de su competencia comunicativa, pudiendo mantener la conversación en español a través de las sesiones, fue constante en una de las participantes. Otros participantes reconocieron haber tenido una mejor fluidez en el diálogo (7 veces). Explicar lo que se quiere decir (5 veces). Esta respuesta comprende aspectos de vocabulario, de formulación de frases y de exposición de un tema.

Pregunta: Mi desempeño en esta conversación fue...

En las apreciaciones de la autovaloración, los estudiantes alemanes dieron sus estimaciones sobre la conversación de la sesión de una manera global. Así, consideraron que su rendimiento personal fue "bueno" (26 veces), "muy bueno" (7 veces), "no muy bueno" (5 veces). Una de las participantes consideró en todas las sesiones que podría mejorar su desempeño.

Pregunta: La(s) actividad(es) que realizaré para mejorar es/son... (corrección).

Las respuestas para esta pregunta fueron escasas. Se mencionaron sobre todo tres tipos de intenciones para mejorar su rendimiento: "aprender el vocabulario de la sesión" (20 veces), "revisar la gramática" (14 veces) y solo una chica mencionó dos veces su propósito de "preparar más detalladamente la próxima sesión".

Se pudo constatar que se mantuvo constante la preocupación por la importancia de aprender y preparar el léxico, adquirir una mayor fluidez en su discurso y la corrección gramatical en las conversaciones, al igual que el uso de las mismas estrategias a lo largo de las sesiones para solucionar los problemas presentados. 


\section{Conclusiones}

Los datos proporcionados en el análisis de los dos diarios revelan aspectos importantes en cuanto al desarrollo del rendimiento del aprendizaje alemán-español en el contexto del teletándem en los centros de lenguas ENALLT-UNAM y el Zentrum für Sprachen Universität Würzburg. Los procesos de autoregulación como el monitoreo personal de destrezas y estrategias de aprendizaje de los estudiantes y del proceso interactivo del diálogo, muestran que durante las conversaciones tándem, los estudiantes evolucionaron de manera bastante positiva llevando a los participantes a la toma de responsabilidad y a la autonomía de su aprendizaje. El aspecto intercultural y la colaboración fueron un gran motor de motivación. Las apreciaciones, en el caso de los estudiantes alemanes, se mantuvieron en un nivel muy alto a este respecto.

Las recensiones finales de los estudiantes alemanes expresan su punto de vista sobre la función del teletándem en los cursos de español en el centro de lenguas de la Universidad de Würzburg. Los comentarios apoyan esta iniciativa porque no solo los puso en contacto con otra cultura para conocer a su gente y sus costumbres, sino también porque fue una buena oportunidad para practicar el español hablando con nativos de la lengua y, así, poder mejorar sus conocimientos de español. También lo ven como un "muy buen" complemento de los cursos de español o como una "continuación de los mismos".

Durante el semestre se constató, en el curso de español, de una manera notable, la confianza que adquirieron en sí mismos, los participantes del teletándem, al expresarse oralmente en español, en comparación con otros compañeros que no participaron en este proyecto. Además, su rendimiento oral fue en aumento en cuanto a que podían encontrar más fácilmente el léxico que necesitaban al expresarse y hablar con mayor fluidez. También el grupo de Teletándem aportó elementos importantes al curso de español como la motivación, la creatividad y la colaboración al momento de abordar temas del curso y el trabajo en equipo.

Se puede decir, finalmente, que el proyecto teletándem entre la Escuela Nacional de Lingüística Aplicada (ENALLT-UNAM) y el Zentrum für Spachen Universität Würzburg es una gran oportunidad para ofrecer a los estudiantes que aprenden español o alemán una alternativa promotora del aprendizaje autónomo que, sin duda, solo puede tener repercusiones positivas en el rendimiento de su aprendizaje. 
- El teletándem en el aprendizaje Alemán - Español: mediateca ENALLT - UNAM y Universidad de Würzburg

\section{Referencias}

BRAMMERTS, H. Tandemberatung. Zeitschrift für Interculturellen Fremdssprachenunterricht ZIF, v. 11, n. 2, p. 1-16, 2006.

CAVALARI, S. M. S.; ARANHA, S. Teletandem: integrating e-learning into the foreign language classroom. Actascieniarum Language und Culture, Maringá, v. 38, p. 327-336, Oct.-Dec. 2016.

CHIAPELLO, S.; GONZÁLEZ ROYO, M.; MURA, A. Teletándem: actividad interactiva del discente de I/LE y su percepción mediante una encuesta de autoevaluación. In: XII Jornadas de Redes de Investigación en Docencia Universitaria. Alicante: Universidad de Alicante, 2014, p. 2079-2095.

CONSEJO DE EUROPA. Marco Común Europeo de Referencia para las Lenguas: Aprendizaje, Enseñanza, Evaluación. (traducido por Instituto Cervantes). Madrid: Anaya, 2002. Disponible en: https://cvc.cervantes.es/ensenanza/biblioteca_ele/marco/. Consulta en: 08 mar. 2018.

GONZÁLEZ, G. Las estrategias de aprendizaje en el alumnado de primer grado como herramienta de mejora. XII Jornadas de Redes de Investigación en Docencia Universitaria. Alicante: Universidad de Alicante, 2014, p. 1448-1449.

LORENZO, A.; DE COULOMB, G. Técnicas para la conversación en la clase de ELE. Instituto Cervantes, 2005. Disponible en: https://cvc.cervantes.es/ensenanza/biblioteca_ele/ publicaciones_centros/PDF/rio_2005/09_Lorenzo-Coulomb.pdf. Consulta en: 08 mar. 2018.

MENDOZA, A. Las interacciones de Teletándem entre la mediateca del CELE-UNAM y la Universidad Nacional Estatal de São Paulo, UNESP, campus Assis, como una experiencia de autonomía. Leaa Lenguas en Aprendizaje Autodirigido. Revista Electrónica de la Mediateca del CELE-UNAM, año 6, n. 1 p. 1-23, 2014.

TARDO, F. Potenciar las estrategias comunicativas en las clases de ELE: una opción viable para desarrollar las habilidades orales. Revista electrónica de didáctica/español lengua extranjera, n. 5, p. 1-7, 2005. 
VASSALLO, M. L.; TELLES, J. Foreing Language Learning in-Tandem: Theoretical Principles and Reasearch Perspectives. Aprendizagem de Línguas Estrangeiras In-Tandem: Princípios Teóricos e Perspectivas de Pesquisa. The ESPecialist, v. 27, n. 1, p. 83-118, 2006.

COMO CITAR ESTE ARTIGO: LECONA, Edú Alberto Cruz; MARTíNEZ, María Guadalupe ALFARO. El teletándem en el aprendizaje Alemán - Español: mediateca ENALLT - UNAM y Universidad de Würzburg. Revista do GEL, v. 15, n. 3, p. 88-108, 2018. Disponível em: https://revistadogel.gel.org.br/

DOI: http://dx.doi.org/10.21165/gel.v15i3.2411

Submetido em: 17/11/2018 | Aceito em: 14/12/2018. 